\title{
Correspondence of Escape-Turning Behavior with Activity of Descending Mechanosensory Interneurons in the Cockroach, Periplaneta americana
}

\author{
Shuping Ye and Christopher M. Comer \\ Neuroscience Group, Department of Biological Sciences, University of Illinois at Chicago, Chicago, Illinois 60607
}

Two bilaterally paired mechanosensory neurons that respond to antennal touch stimulation recently have been described in the cockroach Periplaneta americana. Here chronic recordings were used to describe the activity of these interneurons in relation to behavior. Parallel intra/extracellular recording experiments showed that both pairs of previously identified descending mechanosensory interneurons (DMIs) were activated after touch stimulation of the antennae and before initiation of escape. On a trial-by-trial basis, the bilateral pattern of their activity was correlated with sensory input and behavior: when one antenna was touched, the contralateral DMI axons displayed impulses earlier and in greater numbers than their ipsilateral homologs; turns were made toward the side with greater
DMI activity, i.e., away from the touched antenna. One parameter of DMI activity (the bilateral difference in number of DMI impulses) was correlated with the angular amplitude of turning. In the absence of touch stimulation, unilateral electrical stimulation of a cervical connective via the chronic electrodes produced turning movements similar to natural escape turning and of appropriate directionality. These results support the hypothesis that neural activity in DMls is involved in the control of antennal touch-evoked escape, and they provide a basis for a model of DMI specification of the direction of escape turning.

Key words: antennae; chronic recording; cockroach; escape behavior; sensory coding; touch
A large body of evidence indicates that, when evasive movements of cockroaches are triggered by wind, information encoded by the bilaterally paired giant interneurons (GIs) determines the direction of the initial turning component of escape (Comer, 1985; Camhi and Levy, 1989). When escape is elicited by touching an antenna, it also begins with a turning movement away from the stimulus (Comer et al., 1994; Stierle et al., 1994). The descending mechanosensory interneurons (and in particular DMIa-1 and DMIb-1) described in the companion paper (this issue, Burdohan and Comer, 1996) should be able to transmit antennal touchsensory information quickly to thoracic motor centers. However, to understand the multisensory control of escape, it is necessary to determine whether the DMIs encode directional information that is expressed in escape behavior and to compare the integration of touch-sensory cues with the integration of wind-sensory information. With a system recently developed for this purpose (Ye et al., 1995), we have recorded from the neck connectives of intact cockroaches during escape so that activity of the DMIs could be tested for correlations with escape movements on a trial-by-trial basis. We especially were interested in two issues: would DMI activity have timing characteristics adequate to explain the very short latency of escape elicited by antennal contact, and could DMI activity explain the directionality of the initial pivot away from a stimulus? We report here that, when the antennae of intact

Received Feb. 21, 1996; revised June 21, 1996; accepted June 25, 1996.

This work was supported by a grant from the National Science Foundation (IBN-9222619) to C.M.C. We thank Jane Roche King for reading an earlier version of this manuscript and S. B. for encouragement.

Correspondence should be addressed to Dr. Christopher M. Comer, Department of Biological Sciences (M/C 066), University of Illinois at Chicago, 845 West Taylor Street, Chicago, IL 60607.

Dr. Ye's present address: Department of Neurology, Emory University School of Medicine, WMB Suite 6000, P.O. Drawer V, Atlanta, GA 30322.

Copyright (C) 1996 Society for Neuroscience $0270-6474 / 96 / 165844-10 \$ 05.00 / 0$ animals were abruptly touched, large-amplitude impulses in the cervical connectives were recorded at short latencies and before the onset of movement. The pattern of activity in the large units on the two sides of the nerve cord predicted both the direction and angular amplitude of evasive turns. Intracellular recordings demonstrated in particular that DMIs a- 1 and b- 1 contribute to this neural activity associated with turning. Electrical stimulation of cervical axons through the recording electrodes produced turning movements that were consistent with the laterality of antennal mechanosensory information in the DMI pathway. These results provide evidence for control of antennal touch-evoked escape by DMIs and provide an initial model for DMI specification of the directionality of escape.

A preliminary description of some portions of this work has been published (Ye and Comer, 1993).

\section{MATERIALS AND METHODS}

All animals were adult male Peripleneta americana. They were either raised in our own colonies or obtained from commercial suppliers. Some preliminary electrophysiological recordings were made with standard extracellular metal hook electrodes, as described in a previous work (Burdohan and Comer, 1990), but most recordings were made with the specialized methods described below.

Simultaneous recording of behavioral and neural activities. Evasive turning and running were recorded with a motion-tracking system (MTS; Fig. 1), the details of which have been reported elsewhere (Ye et al., 1995). Briefly, an animal had a support bar attached to its pronotum, and the animal was placed on the apex of a hollow Styrofoam sphere. The sphere, supported by an air-floated ball bearing, rotated passively with little friction as the animal ran. Rotation of the sphere was transduced into electrical signals by a pair of shaft-angle encoders (Fig. 1, Ex, Ey) that contacted the equator of the sphere by way of light plastic wheels. Specially developed computer hardware and software were used to digitize the encoder signals, compute the motion of the sphere, and then reconstruct the animal's intended movements.

Three aspects of escape behavior could be derived from MTS record- 


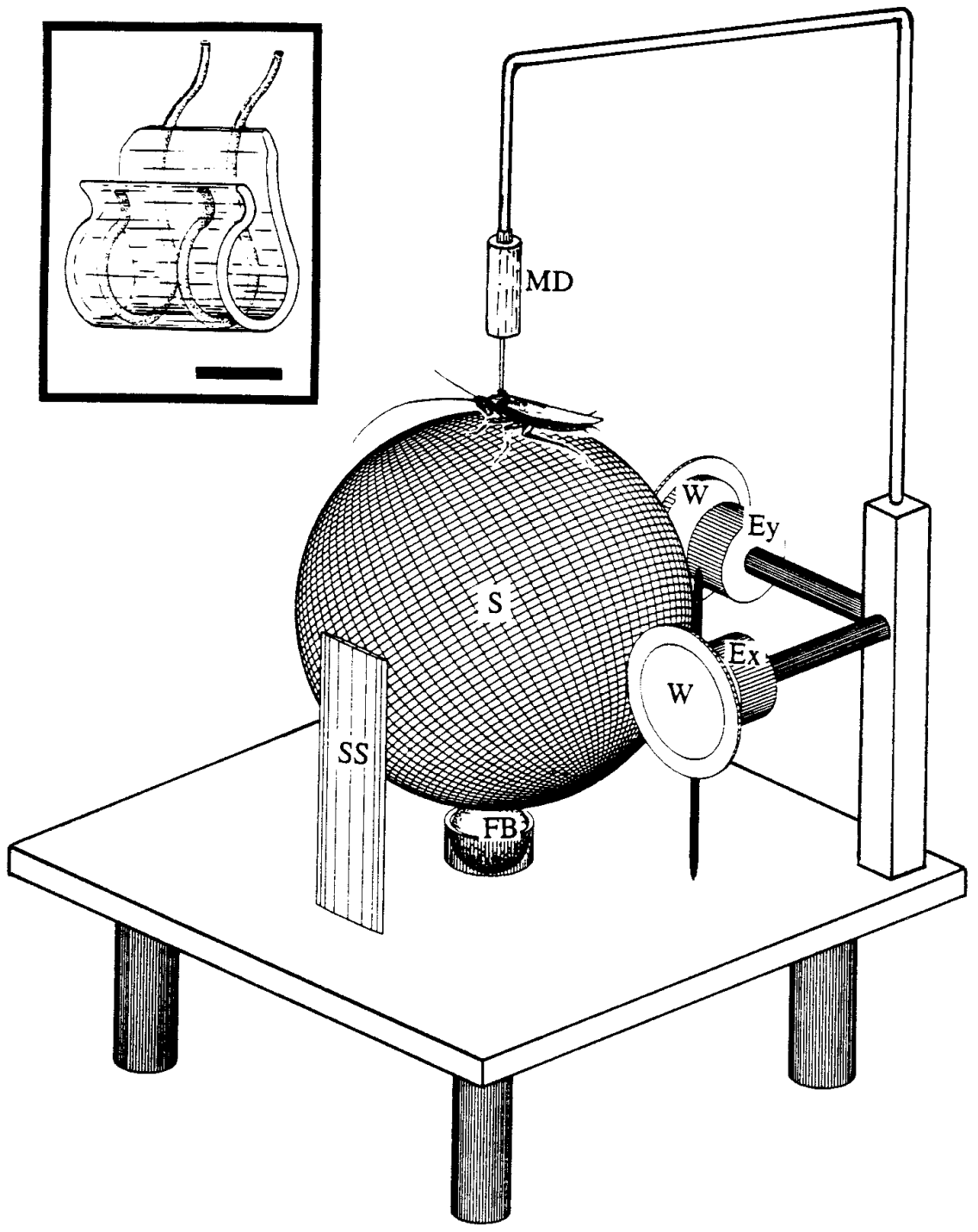

Figure 1. Setup for tracking escape behavior while making chronic recordings of neural activity. Drawings are to scale. $E x, E y$, Optical shaft-position encoders; $F B$, air-floated ball bearing (air piped in from below); $M D$, mounting device; $S$, hollow Styrofoam sphere (6 $\mathrm{gm} ; 12 \mathrm{~cm}$ in diameter); $S S$, supporting glass slide (another is visible to the right in the side view; a third is behind the sphere); $W$, plastic wheel. Inset, Clip electrode; scale bar (applies to inset only), $100 \mu \mathrm{m}$. ings: (1) the escape latency, which was the time (at a $1 \mathrm{msec}$ resolution) between the onset of the stimulus and the first movement made by the animal; (2) the angle of an escape turn, which corresponded to the difference between an animal's orientation before the stimulus was delivered and its orientation at the end of the initial pivot (using a time criterion as in previous work with both free-ranging and tethered animals; Ye et al., 1995); (3) the escape trajectory, which depicted the change of the animal's position over time for the entire escape run (see Fig. 2). The present analysis considers only timing and directionality of the initial turn.

Specially designed cuff electrodes, which we refer to as clip electrodes, were used to record neural activity from the cervical connectives of behaving animals. For each animal, we used two electrodes, one for each hemiconnective. A single-clip electrode (Fig. 1, inset) was composed of two conducting wires ( 25 or $50 \mu \mathrm{m}$ insulated nichrome). One end of each wire was embedded in an omega-shaped epoxy clip. On the inner surface of the clip, the epoxy and the insulation of the wire were removed so that a $100 \mu \mathrm{m}$ length of wire opposite to the clip opening was exposed.

For implantation of electrodes, an animal was cooled at $4^{\circ} \mathrm{C}$ until movement ceased. Then it was positioned ventral side up (with legs restrained) on a wax block, and a longitudinal incision was made in the ventral cuticle of the neck. Two clip electrodes were cemented together and lowered between the connectives with the aid of a micromanipulator. Each hemiconnective was placed into a clip opening. After completing placement of electrodes, we returned the flaps of cuticle to their original position and used clotted hemolymph to seal the incision. The electrode leads emerging from the incision were waxed to the cuticle at several points and brought to the edge of the pronotum.
After implantation, animals were mounted in the MTS and allowed at least $1 \mathrm{hr}$ to recover. Most animals (75\%) were walking vigorously, actively moving antennae, and occasionally grooming well before $1 \mathrm{hr}$ had elapsed. Animals that were not active by this time were excluded from further study.

Relationship between overall neural activity and DMI activity. Simultaneous intra- and extracellular recording was used to investigate the relationship between overall neural activity and impulses of individual cells in the cervical connectives. In these parallel experiments we maintained extracellular recording conditions nearly identical to those in the behaving animals. These animals did not have the body cavity dissected. Their legs were removed, and pins (that did not pierce the body) were used to hold them to the substrate. The only incision was in the neck for implanting electrodes, as described above. For this part of the study, we manufactured clip electrodes with a longer shaft, so they could be held in place with a micromanipulator. The clip assembly then served as the platform stabilizing the cord during intracellular penetration. Axons within the cervical connectives were impaled with glass micropipettes filled at the tip with $4 \%$ Lucifer yellow (tip resistances, 50-100 $\mathrm{M} \Omega$ ). After the completion of recording, Lucifer yellow was injected by using 3-5 nA of hyperpolarizing current. The nerve cord and brain were then extracted, fixed, and cleared according to standard methods (Westin et al., 1988). Whole mounts of the rostral ganglia were examined under epifluorescent illumination to establish the identity of filled cells. We focused on the two descending mechanosensory interneurons (DMIa-1 and DMIb-1) with the largest cervical axons (Burdohan and Comer, 1996).

Sensory stimulation. Turning and running behavior was elicited in these 
Figure 2. Tapping an antenna elicits short-latency contraversive turning and running. Top, Two original experimental records from locomotor tracking system. Animal (symbolized with icon in center to show orientation; circle, head; dotted line, anterior-posterior axis; short diverging lines, cerci) was free to turn within $360^{\circ}$ frame of reference. When animal ran, its intended movement was plotted to give the trajectories shown. Left, Animal was tapped on right antenna-turned left and ran. Right, Animal was tapped on left antenna-turned right and ran. Bottom, Circular histogram gives initial angle of turn for 215 trials from 11 animals (data from trials on which right antenna was stimulated were normalized so they could be plotted with trials on which left antenna was stimulated). Large arrow shows angle of mean vector for turning (Theta $=118.6^{\circ} ; 95 \%$ confidence interval $=112$ $\left.126^{\circ}\right)$. Standard histogram displays distribution of latencies (in milliseconds) for these 215 trials. Arrow at top represents the mean latency $(24.5 \mathrm{msec}$; $95 \%$ confidence interval $=22-27 \mathrm{msec}$ ).
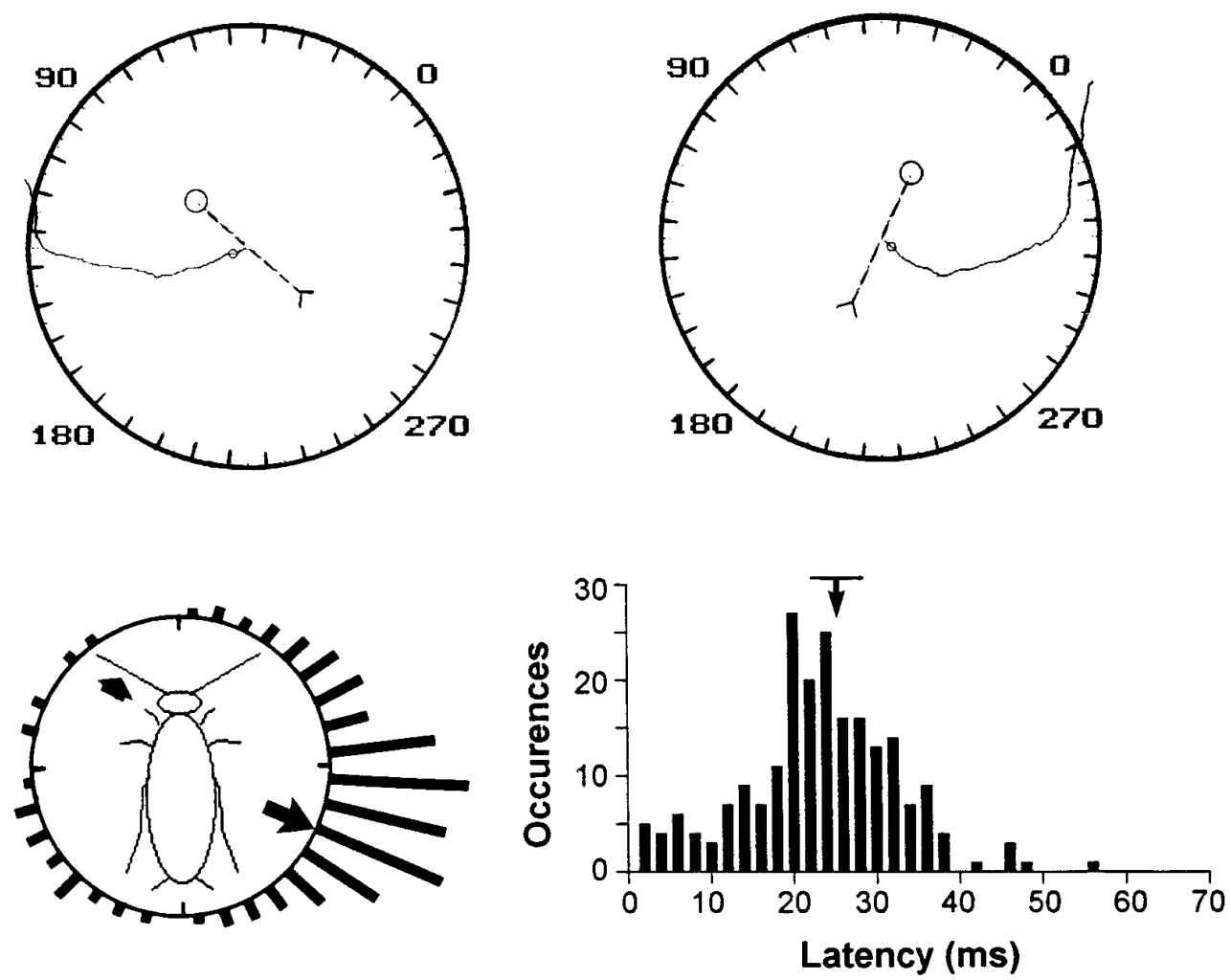

experiments by moving a probe to contact one antennal flagellum abruptly. Because this requires moving an object in front of and quite near an animal, it is important to note that evasive behavior evoked in this way depends only on processing of antennal touch-sensory information. Blocking vision or cercal wind receptors does not prevent responses to touch (Comer et al., 1994; S. Ye and C. Comer, unpublished observations).

A replicable contact stimulus was generated with a solenoid-driven probe fabricated in our lab. Details of its construction have been described (Ye et al., 1995). Briefly, the probe was a bar $8 \mathrm{~cm} \times 2 \mathrm{~mm}$ in diameter; it could rotate about its base, which was mounted in a plastic handle. A solenoid in the handle was activated to displace the bar abruptly at a fixed velocity $(3 \mathrm{~m} / \mathrm{sec})$. The range of the displacement was adjustable. A $2 \mathrm{~cm}$ displacement (as measured at the tip of the probe) was used for contacting an antenna. To stimulate an animal, we first brought the probe slowly to the vicinity of the animal and positioned it midway between the base and the tip of the antennal flagellum. The stimulus was activated when the distance between the probe and the antenna was approximately one-half the range of the probe swing, i.e., $\sim 1 \mathrm{~cm}$ from an antenna. At the time the solenoid was activated to move the probe, a sync pulse was produced to trigger collection of both behavioral and neural data by a computer. The direction in which the touch probe moved was always at a right angle to the long axis of the antennal flagellum, and it was displaced from lateral to medial. The exact position of the antennal flagellum (with respect to the head) at the time of stimulation was not controlled rigidly. However, stimuli were applied only when the flagellum was held within a "standard" range: from $0^{\circ}$ (pointing directly forward and parallel to the antero-posterior axis of the head) to $90^{\circ}$ (pointing directly to the side of the head). Each flagellum can be moved actively to point across the antero-posterior axis of the head to the opposite sensory field or into the sensory field on the same side but to the rear of the head, but stimuli were not applied when it was in these extreme positions.

Electrical stimulation. For electrical stimulation of a cervical connective, the two leads from each clip electrode were connected to the output of an electronic stimulator with a stimulus isolation unit (WPI A-310, with A-360 SIU). The voltage level and pulse frequency of stimulation were varied, as indicated in Results, but the total duration of stimulation (pulse train length) was fixed at $300 \mathrm{msec}$.

Data analysis. When extracellular records were analyzed, our software counted waveforms as "large-amplitude" action potentials if they met the following criteria: (1) they were $>75 \%$ of the maximal peak-to-peak amplitude observed, and (2) they were not $>2 \mathrm{msec}$ in duration. This size criterion is more stringent than that used in initial studies of the DMI population (Burdohan and Comer, 1996). Here we wished to focus our recordings more specifically on the two very largest cervical axons, those of DMIa-1 and DMIb-1. (From our correlated intracellular recordings, we know that the criterion used captured better than $90 \%$ of all DMIa-1 impulses and $\sim 50 \%$ of those from DMIb-1. Using a less stringent amplitude criterion would have capture more DMIb-1 impulses but also would have captured many impulses not belonging to either of the two largest DMIs.) In most cases we counted all of these events that occurred within $50 \mathrm{msec}$ of stimulus onset. However, in one case we used a $70 \mathrm{msec}$ counting bin to facilitate comparisons with previous studies (Burdohan and Comer, 1996; see below).

When averages are reported, they are always given as the mean \pm SEM. The results from trials in which large-amplitude impulses were related to turn angles were analyzed by using the Pearson productmoment correlation (Sokal and Rohlf, 1981).

\section{RESULTS}

\section{Baseline for touch-evoked turning behavior}

Animals mounted in the MTS generate escape behavior similar to that seen in animals observed under completely free-ranging conditions (Ye et al., 1995). Figure 2 summarizes the timing and directionality of the evasive behavior elicited in this study by contact stimulation of the antennae (these animals did not have implanted recording electrodes). As seen at the top of the figure, the direction of the initial turn depended on which antenna was stimulated: animals turned away from the side on which the antenna was tapped. At the bottom of Figure 2, over 200 trials from 11 different animals are summarized in a circular histogram to illustrate the directionality of escape turning in response to touching one antenna. Responses to touching the right antenna were normalized and coplotted with responses to touching the left antenna. Of all responses, $87.6 \%$ were contraversive with respect to the stimulated antenna. The mean vector of turning was $119^{\circ}$ $\left[95 \%\right.$ confidence interval $=112-126^{\circ}$; mean vector significantly 
different from $0^{\circ}$ at $p>0.01$ level (Batschelet, 1981)]. On these same trials, response latency varied from 2 to $56 \mathrm{msec}$, with an average value of $24.5 \pm 1 \mathrm{msec}(95 \%$ confidence interval $=$ 22.6-26.4 msec). Thus, the physiological recordings obtained here could be correlated with behavior that was of appropriately short latency and that consisted of turns directed away from the stimulus.

\section{Descending impulse activity recorded under different conditions}

Large-amplitude impulses were recorded readily at the cervical level after a tap to one antenna, and they demonstrated a pattern of lateralization. Figure 3 shows representative extracellular records from animals recorded in a conventional manner: legs and wings removed, eviscerated, pinned to the substrate, and a silver wire hook electrode around each cervical hemiconnective. It is easy to see that touching one antenna gave rise to neural activity, which began at short latencies (7-10 msec after the stimulus), and that included both small- and large-amplitude units (units counted as "large-amplitude" are marked with dots). It is also clear that large-amplitude units were recorded preferentially from the connective contralateral to the antenna that had been stimulated. This same pattern was observed in all experiments conducted in this way ( $n=6$ animals).

In recordings from intact animals, large-amplitude unit activity was recorded at similarly short latencies, but the pattern of lateralization was not always immediately apparent. This was attributable to the fact that, when clip electrodes were implanted for recording from alert, behaving animals, there was considerably more neural activity. This was true both of spontaneous activity and of activity evoked by antennal stimulation (compare Figs. 3, 4). Therefore, a large sample of recordings from behaving animals was analyzed to determine whether lateralization of touch-evoked neural activity typically was present. Counts were made of the number of waveforms recorded from each hemiconnective meeting the criteria for "large-amplitude impulses" (see Materials and Methods). The counting bin encompassed $50 \mathrm{msec}$ after touch stimulus onset. For each trial, we computed the "excess impulses," or the number of events counted from the connective contralateral to the stimulated antenna minus the number from the ipsilateral connective. Any trial yielding a positive number thus would indicate a bias toward more activity on the contralateral side. The results are displayed as Figure 5.

There was indeed a strong bias in the data, with more impulses being recorded from the side of the nerve cord contralateral to the stimulated antenna on $90 \%$ of the trials. The average number of "excess impulses" was $4.4 \pm 0.4$ (the total number of touchevoked large impulses on the contralateral side averaged $7.6 \pm$ 0.5 ; on the ipsilateral side it averaged $3.8 \pm 0.4$ ). In addition to this difference in amount of activity, there was also a consistent bilateral difference in timing: the large-amplitude impulse activity showed up first at the electrode contralateral to the stimulated antenna on all but 1 of the 88 trials analyzed. The latency to appearance of the first large-amplitude impulse on the contralateral side averaged $8.2 \pm 0.7 \mathrm{msec}$ after stimulus onset, and the average on the ipsilateral side was $19.1 \pm 2.0 \mathrm{msec}$. In this set of experiments, the beginning of movement occurred at a mean of $39 \pm 1.7 \mathrm{msec}$ after stimulus onset. These observations establish that, in intact animals, large-amplitude cervical impulse activity arises before the onset of escape movements and that there are two elements of bilateral patterning in this activity. The activity arises earlier in the connective contralateral to the stimulated

\section{Touch Right Ant}

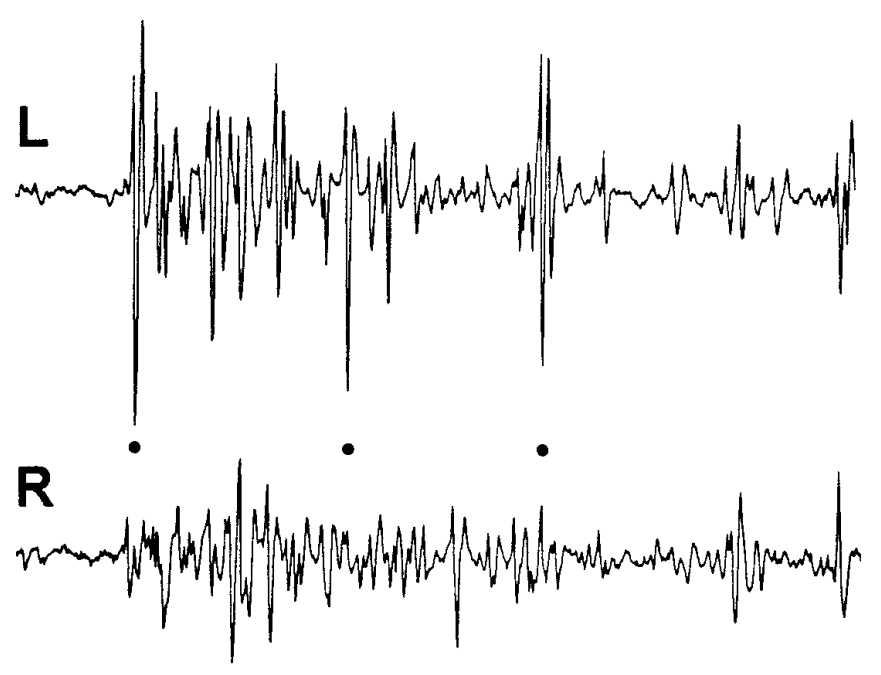

\section{Touch Left Ant}
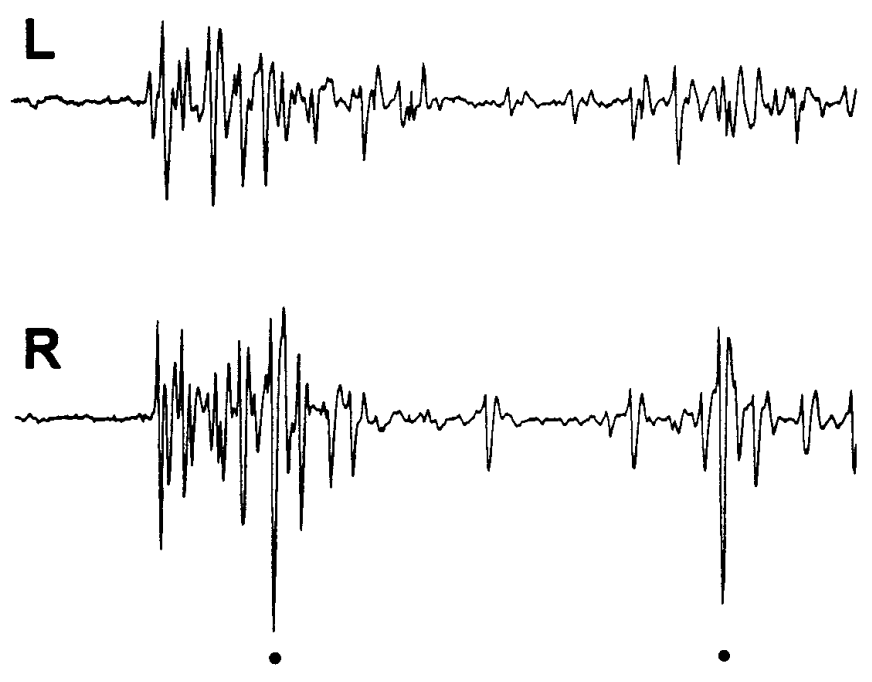

Figure 3. After tapping one antenna, we recorded large-amplitude impulses at the cervical level. Animal was dissected as described in the text. Simultaneous recordings were made with standard metal hook microelectrodes from the left $(L)$ and right $(R)$ cervical connectives. Impulses counted as "large-amplitude" are marked with dots below each trace. Note distinct predominance of large impulses contralateral to the stimulated antenna. Calibration: $0.8 \mathrm{mV}, 10 \mathrm{msec}$.

antenna, and the number of impulses is almost always greater on that side.

At the cellular level, these observations could be interpreted in several different ways. They could mean that, on a given side of the nerve cord, the axons of one or more rapidly conducting interneurons are present and respond to stimulation of both antennae but more strongly to the antenna on the contralateral side. Alternatively, they could indicate that, on a given side of the cord, there are axons of interneurons, such as DMIa-1 (Burdohan and Comer, 1996), responding only to the contralateral antenna. These two explanations are not mutually exclusive. 


\section{Touch Right Ant}
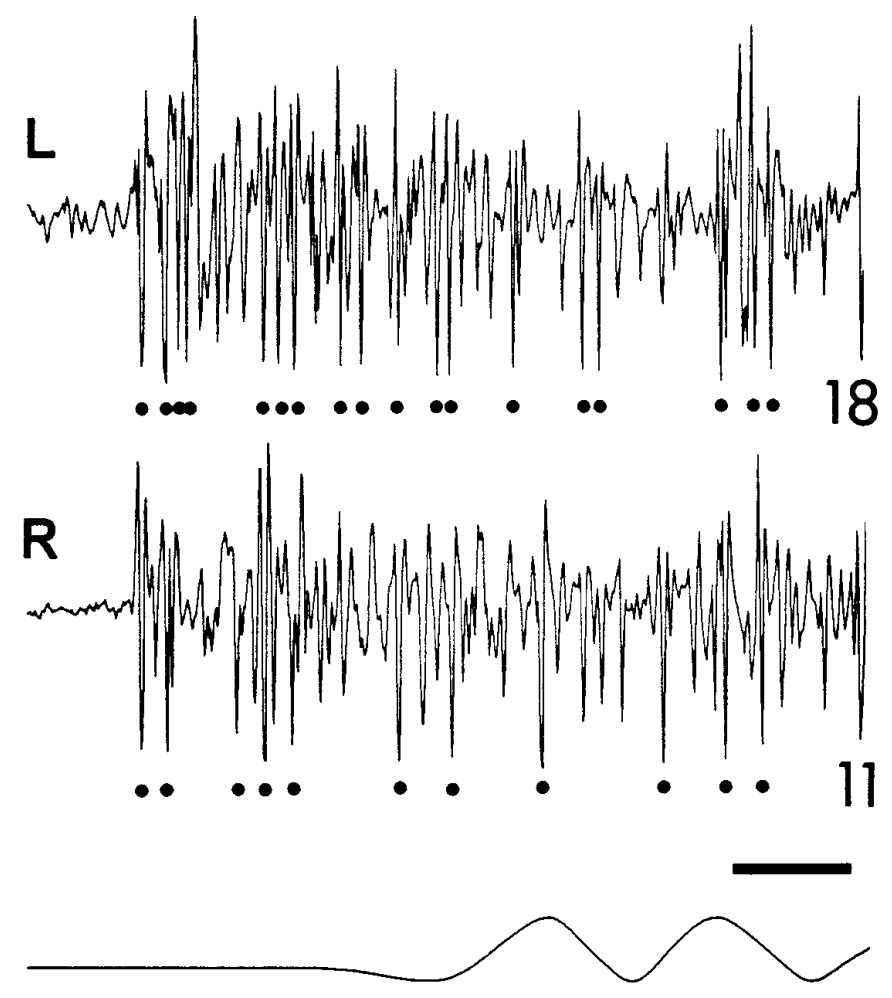

E

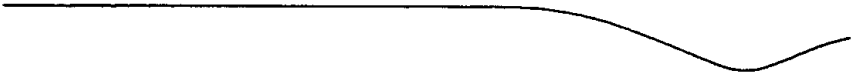

Figure 4. Antennal-driven activity recorded from the cervical level of intact animal. Clip electrodes were implanted around both the left $(L)$ and right $(R)$ cervical connectives. Animal then was placed in locomotion tracking system; output of encoder wheels is shown $(E)$ to indicate that large-amplitude activity preceded the onset of movement. Note that there is more evoked activity overall, so that lateralized pattern of impulses is harder to discern. Impulses counted as "large-amplitude" are marked with dots below each trace, and cumulative count for each is given to the right. Scale bar, $10 \mathrm{msec}$

\section{Identity of large-amplitude impulses}

Simultaneous recordings from the clip electrodes and from intracellular electrodes in minimally dissected animals were accomplished in $>30$ experiments. In 11 of these experiments, the impaled cell was characterized physiologically and completely filled so that it could be identified anatomically. In six cases, DMIs a-1 or b-1 were studied (see below), and in five cases other DMIs were labeled (these will be reported elsewhere; J. Burdohan, S. Ye, and C. Comer, unpublished observations).

As seen in previous work, DMIs a- 1 and b- 1 began firing impulses at short latencies after antennal stimulation. Furthermore, in all cases it was clear that they contributed to the early part of the extracellularly recorded activity, and this was particularly pronounced for a-1 (see below). Another property of these two DMIs noted here was that, just as extracellular recordings from intact animals revealed more overall activity than those from dissected animals (see above), intracellular recordings from DMIs a-1 and b-1 in these minimally dissected animals displayed more

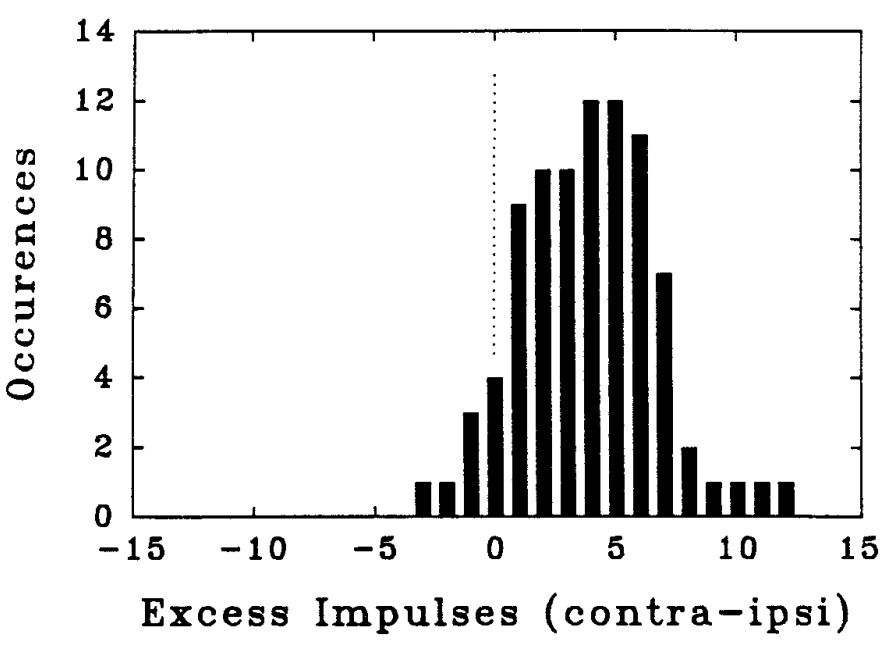

Figure 5. Large-amplitude antennal-driven activity recorded from intact animals is lateralized. Summary of antennal stimulation experiments with six animals. In each trial, the number of large-amplitude impulses recorded from the ipsilateral connective was subtracted from the number recorded at the contralateral connective. This provided a measure of "excess impulses," in which positive or negative values would indicate a bias toward more impulses on the contralateral (contra) or ipsilateral (ipsi) sides, respectively. Of 88 trials summarized, $79(90 \%)$ had positive values, indicating a clear bias toward the contralateral side.

activity than was seen in previous recordings from heavily dissected preparations. This can be seen in Figure 6, in which DMIa-1 fired four impulses after touch to one antenna. In dissected animals, with the basal antennal segments partially restrained, each DMI rarely fired more than two spikes (Burdohan and Comer, 1996). In these minimally dissected preparations, the initial burst of spikes in identified DMIs ranged from 1 to 13 spikes, with a mean of $4.3(n=65$ trials, using the same counting bin, $70 \mathrm{msec}$, as that in the previous study).

DMIa-1 responded only to stimulation of the antenna contralateral to the connective in which its axon was impaled, and its activity was always correlated one for one with the very earliest and very largest amplitude cervical spikes (Fig. 6). Impulses recorded from DMIb-1 were always correlated with large-amplitude units in the extracellular record (but typically not so large as a-1). Although b-1 impulses usually were part of the initial burst of unit activity (Fig. 7), they did not consistently lead off the burst, as was true for a-1. Also, b-1 differed from a-1 because it responded to stimulation of both antennae: either ipsilateral or contralateral to the impaled axon. It would be interesting to know whether there are differences in the way b-1 encodes information about each of the two antennae, because it might reveal contributions of b- 1 to the laterality in the descending pathway attributable to a-1. However, our sample size in this experiment reported here (two b-1 sec recorded and completely filled; 22 total trials) is not large enough to make any definite statements. This point will be documented in detail elsewhere (J. Burdohan, S. Ye, and C. Comer, unpublished observations).

\section{Patterning of DMI impulse activity and turning behavior}

If the bilateral pattern of activity arising from DMIs a- 1 and b-1 provides an animal with some indication of the site of antennal mechanosensory stimulation, then not only should differences in evoked activity in the two cervical connectives be related to which antenna has been stimulated (as shown above), but they should be 


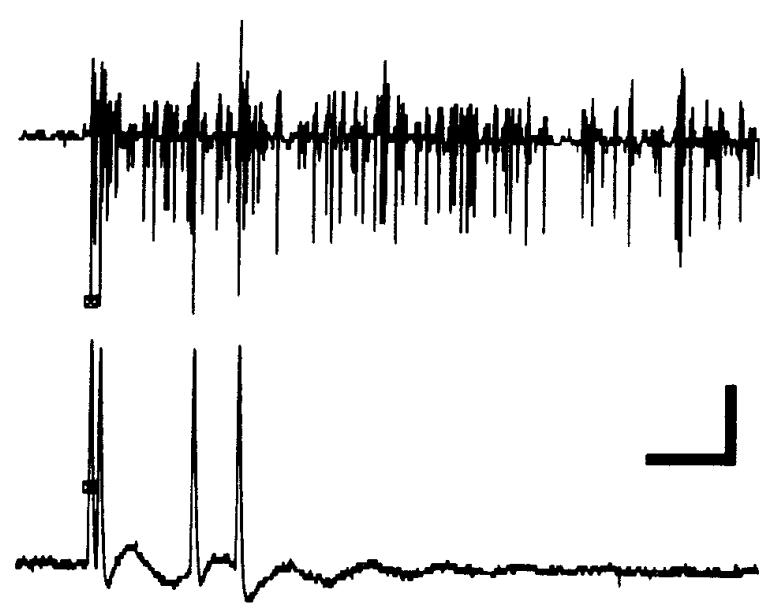

DMI a - 1

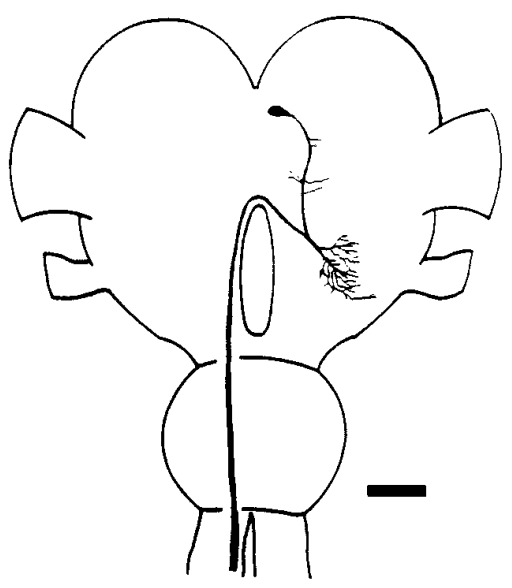

Figure 6. The earliest large-amplitude units in extracellular records correspond to impulses in DMIa-1. Simultaneous extracellular (top) and intracellular (bottom) records are shown. Traces were recorded from one cervical connective while tapping the contralateral antenna. Calibration, $0.2 \mathrm{mV}$ (extracellular), $10 \mathrm{mV}$ (intracellular); $20 \mathrm{msec}$. Drawing below traces is camera lucida reconstruction of cell, the record of which is shown above. Cell (DMIa-1) was filled with Lucifer yellow. Dorsal view of supraand subesophageal ganglia. Scale bar, $200 \mu \mathrm{m}$.

related predictably to the spatial orientation of an animal's turns. Simultaneous behavioral and electrophysiological records obtained from five different animals ( $n=63$ trials) were examined for a correlation between the laterality of touch-evoked largeamplitude activity and the direction of the resultant turn. Because it was unclear a priori which parameter of impulse activity (bilateral differences in timing or number of impulses) would be related to turn orientation, a measure of each parameter was examined.

In all 63 trials, animals turned contraversively with respect to the antenna that was touched. In 62 of the 63 trials, any latency difference that could be measured favored the contralateral side, i.e., the time in milliseconds to the first large-amplitude impulse was shorter at the connective contralateral to the stimulated antenna. That is, in almost all cases animals turned toward the side on which the first large-amplitude impulse was counted. Numbers of impulses were not related quite so closely to the fundamental direction of turn: in 59 of the 63 trials, there were more large- amplitude impulses counted from the connective contralateral to the stimulated antenna (and hence turns toward the side on which the nerve cord displayed more large-amplitude activity). That means that in four cases ( 3 of which were from the same animal) turns were made contraversively with respect to the stimulated antenna but away from the side on which the cord displayed more large impulses. In these cases, the difference was no more than three spikes over the total counting period $(50 \mathrm{msec})$.

Besides the fact that an animal's direction of turn usually could be predicted from a bilateral comparison of descending activity, the particular azimuthal angle of turn also was related to patterns of descending interneuron activity. The magnitude of bilateral latency differences was not related to turn angle (Fig. 8, top; $r=$ $0.1, p>0.05)$. In contrast, differences in the number of largeamplitude impulses on the two sides of the nerve cord were related to turn angle (Fig. 8, bottom). Because there were more large (DMI) impulses recorded from one cervical connective, there tended to be a larger angle of turn to that side $(r=0.4, p<0.001)$.
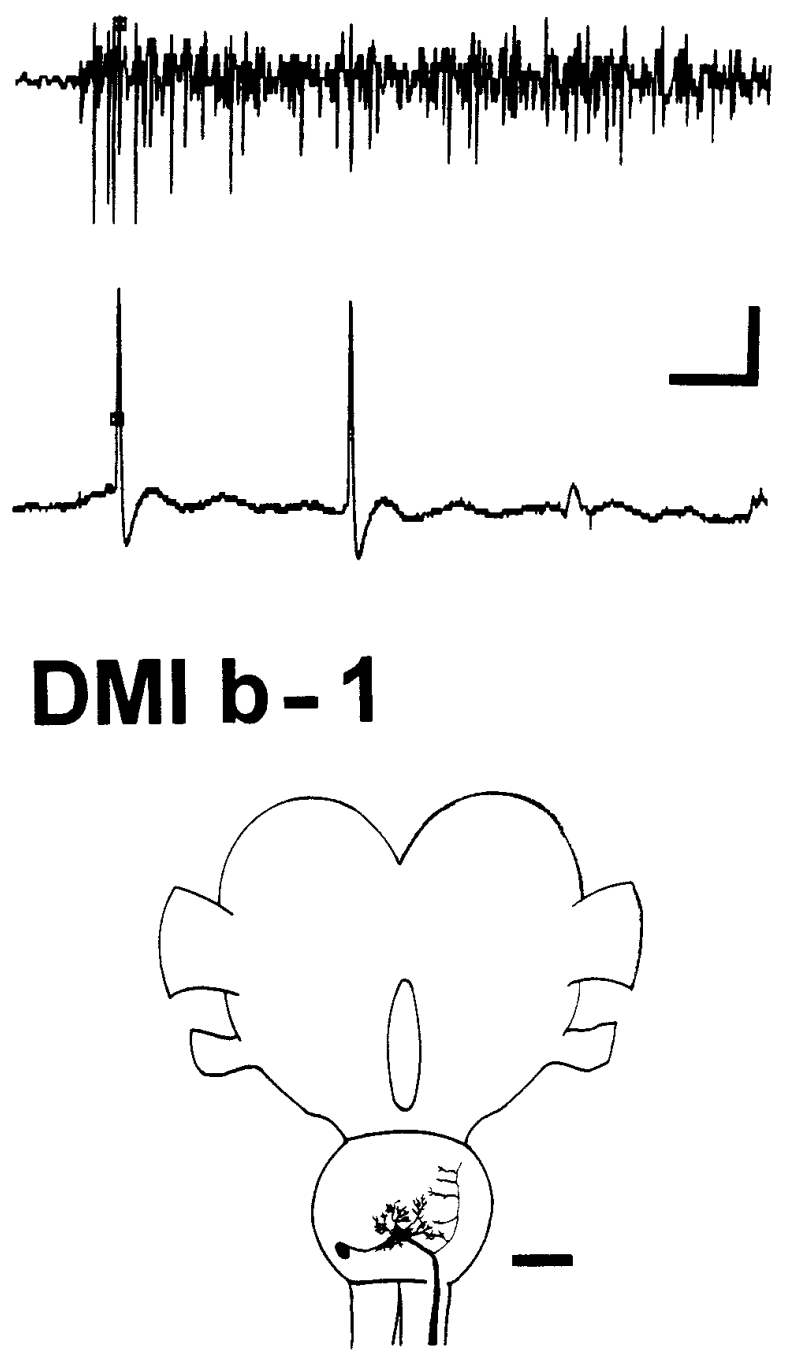

Figure 7. Other large-amplitude units activated by antennal tapping correspond to impulses in DMIb-1. Simultaneous extracellular (top) and intracellular (bottom) records are shown. Traces were recorded from one cervical connective while tapping the contralateral antenna. Calibration: $0.2 \mathrm{mV}$ (extracellular), $10 \mathrm{mV}$ (intracellular); $20 \mathrm{msec}$. Drawing below traces is camera lucida reconstruction of cell, the record of which is shown above. Cell (DMIb-1) was filled with Lucifer yellow. Dorsal view of supraand subesophageal ganglia. Scale bar, $200 \mu \mathrm{m}$. 

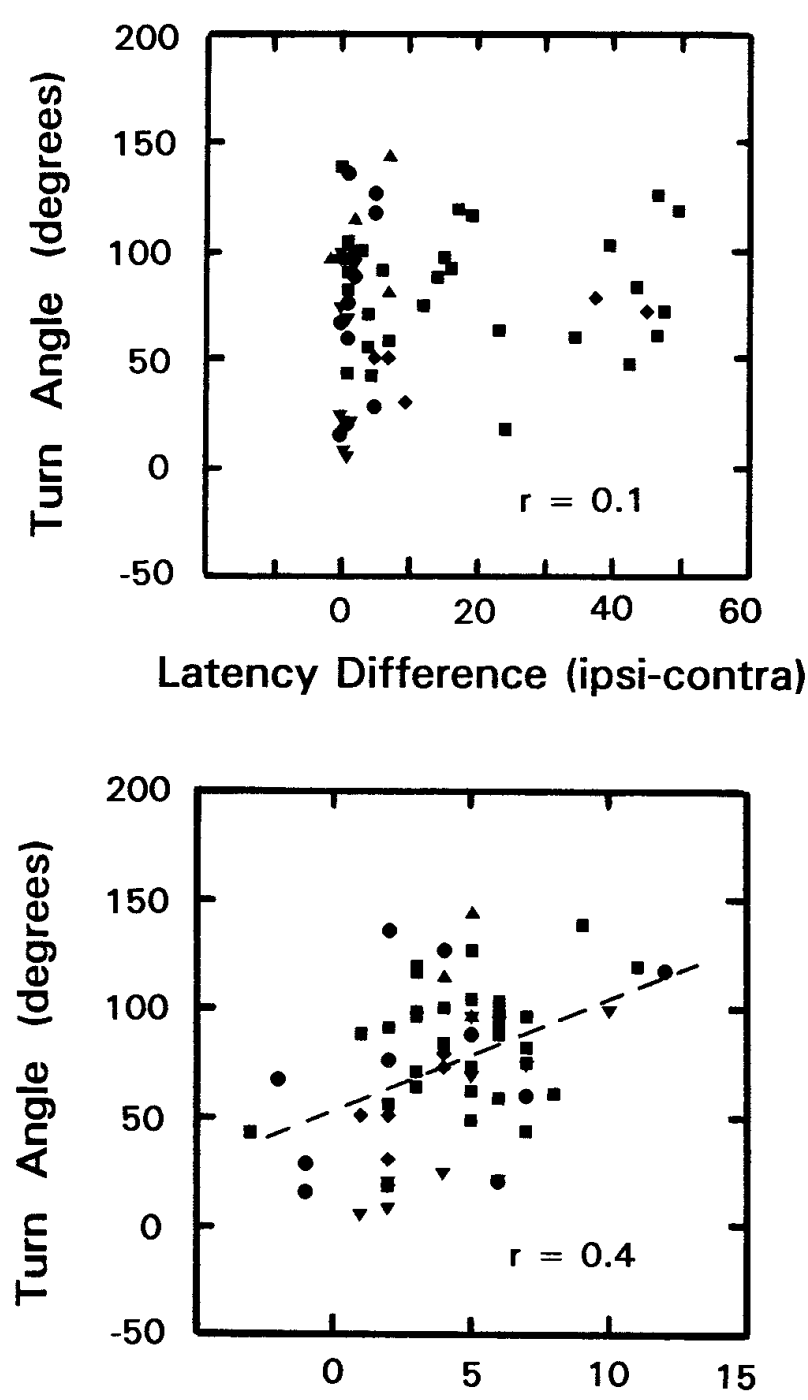

Excess Impulses (contra-ipsi)

Figure 8. Bilateral patterns in timing or number of large-amplitude (DMI) impulses differed in their relationship to angle of turn. Scatter plots give trial-by-trial relationship between observed angle of turn and timing and impulse number. Top, Timing (bilateral differences in latency to first impulse). Bottom, Impulse number (bilateral differences in spike counts or excess impulses, as defined in Fig. 5 and text). Each plot summarizes 63 trials from five animals (each animal's data plotted with a different symbol). Relationship between relative latency and turn angle: $r=0.1$, not statistically significant. However, as number of large-amplitude (DMI) impulses became relatively greater at the contralateral cervical connective, larger initial angles of turn were produced; $r=0.4, p<0.001$.

\section{Turning evoked by cervical electrical stimulation}

If escape is related to impulses in the largest DMIs, then it should be possible to elicit escape turning directly by stimulating the axons of the DMIs in the cervical connective, and turns should be directed toward the side of the stimulated connective. In three different animals, a voltage was applied across the two leads to one of the clip electrodes (to stimulate the connective), and any subsequent behavior was recorded. All three animals responded behaviorally once a voltage threshold was reached (the level of the threshold was between 3 and $6 \mathrm{~V}$ ). In every trial in which the voltage threshold for ANY movement was reached, there was a behavioral response that consisted of a turning movement.

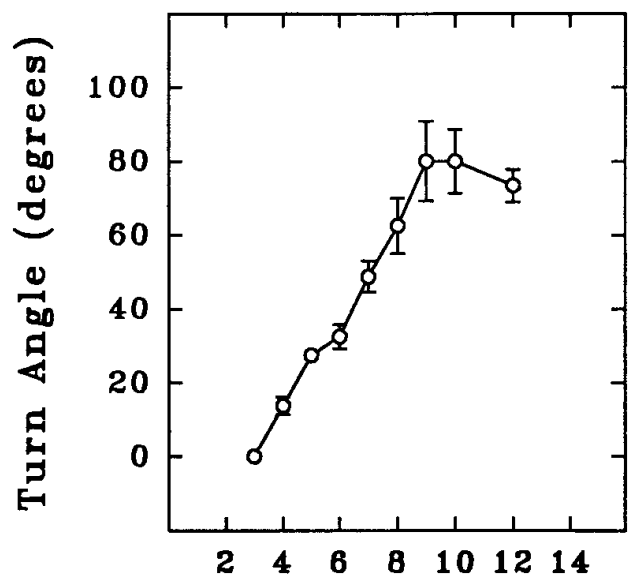

\section{Stimulus Voltage (V)}

Figure 9. Turns can be elicited by electrical stimulation of a cervical connective. Representative data from one animal. Clip electrodes were implanted around both cervical connectives, but only one side was stimulated. All turns were ipsiversive with respect to the side of the CNS that was stimulated. Each point plotted represents the mean of 4-11 trials. Details of stimulation conditions are given in the text.

The direction of the turn was always related to which electrode (connective) was stimulated. All responses from stimulating the right connective were right turns, and all responses from stimulating the left connective were left turns. This is illustrated with data from one of the animals in Figure 9. All turns were directed ipsiversive to the side of the nerve cord that was stimulated $(n=$ 56 total trials), and as stimulus voltage was increased, the average angle of turn increased in a monotonic manner, up to a maximum of $\sim 80^{\circ}$. Beyond this level of stimulation, there was no further increase in turn angle. When trains of pulses were used, the turn angle also increased as the frequency of stimulation increased. The same pattern of turning was seen in both other animals tested in this way.

The production of ipsiversive turns after direct stimulation of one cervical connective fits with the laterality of activity evoked by touch in the descending antennal mechanosensory pathway. Tapping one antenna causes greater DMI impulse activity in the connective contralateral to that antenna, and the greater impulse activity on that side is correlated with a turn ipsiversive to the active side (Fig. 10).

\section{DISCUSSION}

There are very few instances in which it has been possible to describe the signaling of specific nerve cells in relation to natural behavioral responses on a trial-by-trial basis. In vertebrates there are a few cases in which the firing of classes of cortical neurons has been correlated with behavioral decisions (Newsome et al., 1990; Salzman and Newsome, 1994) or directional motor outputs (Georgopoulos et al., 1986). The Mauthner neurons of the medulla and associated spinal circuitry also have provided data of this sort, because they are sufficiently large that their activity can be discerned from extracellular records or with optical monitoring techniques (Nissanov et al., 1990; Fetcho and O'Malley, 1995). However, the possibilities in tethered insects are particularly rich, because they allow for some intracellular recording and/or the establishment of data sets from individually identifiable cells 


\section{Touch Antenna}

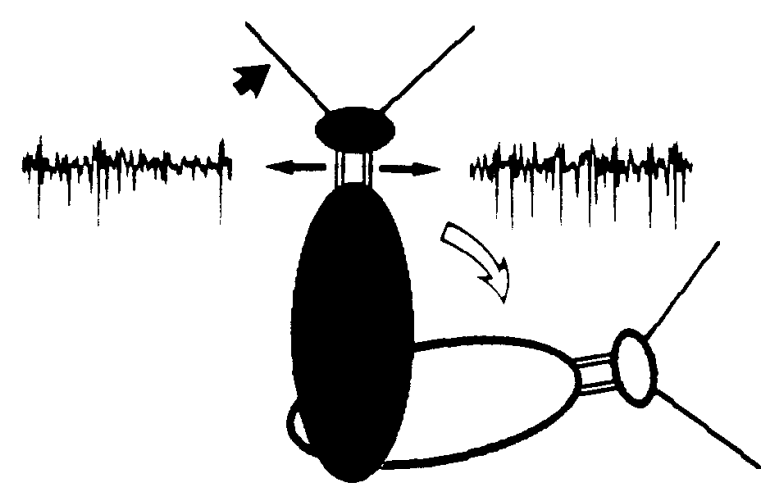

Nerve Stimulation

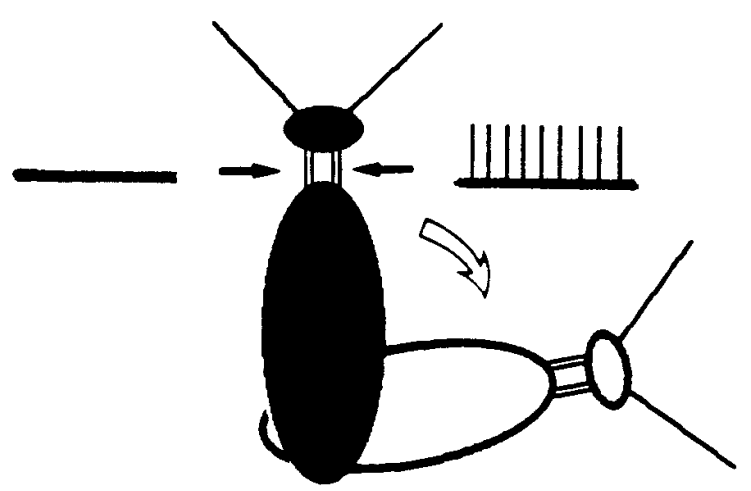

Figure 10. Summary of relation between cervical activity and turn direction. Schematic illustration shows that, when one antenna was touched, there were more large-amplitude (DMI) impulses within the contralateral cervical connective, and the animal usually turned toward that side. Likewise, when activity was evoked electrically on one side only, animals reliably turned toward that side.

(Ramirez, 1988; Böhm and Schildberger, 1992; Hörner, 1992; Kohstall-Schnell and Gras, 1994).

\section{A descending "giant fiber" system for touch-evoked escape behavior}

From the companion anatomical and physiological studies (Burdohan and Comer, 1996) it is clear that DMIs a-1 and b-1 are not the only descending mechanosensory interneurons of cockroaches. However, the reason for focusing on these two DMIs with uniquely large-caliber descending axons was the likelihood that they would (1) be relatively easy to monitor from chronic recording electrodes and (2) show meaningful correlations with behavior on the basis of "first principles" of neuroethology.

First, The intracellular studies reported here confirmed the expectation that impulses of a- 1 and b- 1 would be observable in extracellular records among the very largest amplitude units (Figs. $6,7)$. Nonetheless, because counts of the large impulses in extracellular records almost certainly included some as yet unidentified interneurons, it is quite striking that we found correlations between this impulse activity and the directionality of escape turning. This suggests that, whereas antennal mechanosensory information may reach thoracic ganglia via a variety of interneurons, escape (as a very short-latency response) may be a dedicated function of at least those DMIs that represent the extreme end of the axon caliber spectrum. This is at least approximately analogous to the situation with Mauthner neurons: they are dedicated to the teleost C-start, yet they are only two of a larger set of reticulospinal control elements for tail flipping.

Second, the correlations of DMI physiology with behavior are consistent with the neuroethological principal that evasive behaviors invariably are associated with "giant fiber" systems (Bullock, 1984). Indeed, DMIs a-1 and b-1 are at least as distinctive in size as the well known giant interneurons associated with the cercal wind-sensory system of cockroaches, crickets, and related insects. The two DMIs may not be involved in escape equally; for example, the different times at which each fired in the burst of touchevoked activity (Figs. 6, 7) suggest the possibility that a-1 is important to early phases of the response, perhaps establishing a bias for the choice of turn direction. The later-arriving b-1 information then might contribute to determining the specific angular amplitude of a turn. Ideas such as these require direct tests, and this might be done by extracting separately the impulses in the extracellular records that are attributable to each DMI to look for behavioral correlations. This can be done, in principle (Smith et al., 1988; Gozani and Miller, 1994), and might be attempted in future work. Selective lesions of each cell are also possible by using single-cell killing techniques (Comer, 1985; Selverston et al., 1985), and this also might allow the influences of each cell to be assessed independently.

\section{Modeling the control of turn orientation}

In $90-95 \%$ of behavioral trials, intact animals turned away from the side on which an antenna had been tapped (Fig. 2). How is this initial direction of turn established? A major outcome of this work was to show that, when one antenna is touched, there is a lateralization of descending impulse activity in the large DMIs, with more impulses occurring (and occurring earlier) contralateral to the stimulated antenna (Fig. 5), and that this neural lateralization is reflected in escape-turning behavior. In initial descriptions of antennal touch-evoked turning (Comer et al., 1994), it was noted that, if one cervical connective is transected, responses elicited by touching the contralateral antenna are misdirected: animals often turn toward rather than away from the stimulus. Turns are not misdirected when the antenna ipsilateral to the lesion is touched. Thus, in agreement with the present findings, unilaterally lesioned animals respond as if descending mechanosensory activity in one connective is interpreted to indicate that the contralateral antenna has been touched, and thus they turn toward the more "active" side of the nerve cord (Fig. 10).

We know from physiological analysis of the two largest DMIs (Burdohan and Comer, 1990, 1996), recordings from touchsensitive thoracic interneurons (Ritzmann and Pollack, 1994), and the present recordings from behaving animals that touch of one antenna activates interneuronal activity on both sides of the nerve cord. This indicates that a bilateral comparison of the relative level of activity on the two sides of the nerve cord is involved in determining the direction in which an animal turns. The idea that the impulse activity in bilateral pairs of interneurons determines the laterality of directional motor outputs probably has some general applicability, because it has been found relevant to neg- 
ative phonotaxis (Nolen and Hoy, 1984; Hoy and Nolen, 1987), positive phonotaxis (Horseman and Huber, 1994; von Helversen and von Helversen, 1995), and orientation to pheromone sources (Olberg, 1983; Kanzaki et al., 1994).

A bilateral comparator is the type of model that already has been used to model the specification of wind-evoked turns on the basis of information in the abdominal giant interneurons (Comer and Dowd, 1987; Camhi, 1988; Dowd and Comer, 1988). However, the thoracic motor circuitry seems to use the laterality of neuronal activity in the GI and DMI systems in different ways. In the antennal touch system, if the DMIs on one side (say the right side) are more active, the animals turn ipsiversively (right; Fig. 10), whereas in the wind system, if the GIs on that side (right) are more active, the animals turn contraversively (left; Camhi and Tom, 1978; Comer and Dowd, 1987). Presumably, either two distinct populations of thoracic premotor cells process the different interneuronal signals, or a set of shared thoracic elements receives inputs from the GI and DMI systems in quite different ways. The laterality of cuticular touch input to thoracic interneurons seems to have the same organization as the GI/wind system (Ritzmann and Pollack, 1994), but the relation of DMI/antennal inputs to wind inputs still needs to be clarified. The results presented here begin to provide some answer to the question of what neural information actually is being compared when DMI activity is integrated. The particular orientation of an escape turn has, of course, two parameters: the turn is either to the left or the right and is directed to some azimuthal angle. The data reported here and by Comer and Dowd (1987) suggest that these two aspects of motor output are not determined by the same neural information, so we will discuss them separately.

Turn direction was related here to both the relative timing of DMI impulses on the two sides of the nerve cord and their relative number. The relationship was slightly stronger for latency (see Results), but our data do not allow us to choose between the two. On the basis of considerations of speed alone (an important consideration in antipredator responses), one might expect the system to begin a motor response by turning toward the side with the earliest DMI activity. In essence, this temporal scheme for choosing turn direction would be similar to that known for the Mauthner system, in which one impulse in a Mauthner cell initiates a C-start tail flip toward the side of the active M-cell axon (Nissanov et al., 1990). However, when the importance of bilateral coding for turn direction was examined for wind-evoked turns mediated by the GIs, differences in latency seemed not to be so important as differences in spike number (Liebenthal et al., 1994).

Unlike turn direction, angle of turn showed a very clear-cut difference in the degree to which timing or impulse number could predict the outcome. The magnitude of the timing difference bore no systematic relationship to the angle of turn, but the difference in number of large (DMI) impulses on the two sides was correlated significantly with the angle of turn observed on each trial (Fig. 8). The importance of bilateral coding parameters for specification of turn angle has not yet been tested directly for windevoked escape mediated by the GIs. However, the role of relative timing versus intensity of descending signals in coding cockroach escape turns can be addressed in the DMI system by examining turns evoked by electrical stimulation of the cervical connectives. Knowing how DMIs and cells of smaller axonal caliber are activated may provide insight into the control of turn metrics, such as which factors determine the maximum angle of turn that can be evoked electrically. Finally, systematically varying the pattern of electrical stimulation to one or both pairs of DMIs and then monitoring resultant turning will allow detailed models of DMI control of escape to be formulated.

\section{REFERENCES}

Batschelet E (1981) Circular statistics in biology. London: Academic.

Böhm H, Schildberger K (1992) Brain neurones involved in the control of walking in the cricket Gryllus bimaculatus. J Exp Biol 166:113-130.

Burdohan JA, Comer CM (1990) An antennal-derived mechanosensory pathway in the cockroach: descending interneurons as a substrate for evasive behavior. Brain Res 535:347-352.

Burdohan JA, Comer CM (1996) Cellular organization of an antennal mechanosensory pathway in the cockroach, Periplaneta americana. J Neurosci 16:5830-5843.

Bullock TH (1984) Comparative neuroethology of startle, rapid escape, and giant fiber-mediated responses. In: Neural mechanisms of startle behavior (Eaton RC, ed), pp 1-13. New York: Plenum.

Camhi JM (1988) Escape behavior in the cockroach: distributed neural processing. Experientia 44:401-408.

Camhi JM, Levy A (1989) The code for stimulus direction in a cell assembly in the cockroach. J Comp Physiol [A] 165:83-97.

Camhi JM, Tom W (1978) The escape behavior of the cockroach Periplaneta americana. I. Turning response to wind puffs. J Comp Physiol [A] 128:193-201.

Comer CM (1985) Analyzing cockroach escape behavior with lesions of individual giant interneurons. Brain Res 335:342-346.

Comer CM, Dowd JP (1987) Escape-turning behavior of the cockroach. Changes in directionality induced by unilateral lesions of the abdominal nervous system. J Comp Physiol [A] 160:571-583.

Comer CM, Mara E, Murphy KA, Getman M, Mungy MC (1994) Multisensory control of escape in the cockroach Periplaneta americana. II. Patterns of touch-evoked behavior. J Comp Physiol [A] 174:13-26.

Dowd JP, Comer CM (1988) The neural basis of orienting behavior: a computational approach to the escape turn of the cockroach. Biol Cybern 60:37-48.

Fetcho JP, O'Malley DM (1995) Visualization of active neural circuitry in the spinal cord of intact zebrafish. J Neurophysiol 73:399-406.

Georgopoulos AP, Schwartz AB, Kettner RE (1986) Neuronal population coding of movement direction. Science 233:1416-1419.

Gozani SN, Miller JP (1994) Optimal discrimination and classification of neuronal action-potential waveforms from multiunit, multichannel recordings using software-based linear filters. IEEE Trans Biomed Eng 41:358-372.

Hörner M (1992) Wind-evoked escape running of the cricket Gryllus bimaculatus. II. Neurophysiological analysis. J Exp Biol 171:215-245.

Horseman G, Huber F (1994) Sound localization in crickets. II. Modeling the role of a simple neural network in the prothoracic ganglion. J Comp Neurol 175:399-413.

Hoy RR, Nolen TG (1987) The role of behavioral context in decisionmaking by an identified interneuron in the cricket. In: Higher brain functions (Wise S, ed), pp 133-155. New York: Wiley.

Kanzaki R, Ikeda A, Shibuya T (1994) Morphological and physiological properties of pheromone-triggered, flip-flopping descending interneurons of the male silkworm moth, Bombyx mori. J Comp Physiol [A] 175:1-14.

Kohstall-Schnell D, Gras H (1994) Activity of giant interneurons and other wind-sensitive elements of the terminal ganglion in the walking cricket. J Exp Biol 193:157-181.

Liebenthal E, Uhlmann O, Camhi JM (1994) Critical parameters of the spike trains in a cell assembly: coding of turn direction by the giant interneurons of the cockroach. J Comp Physiol [A] 174:281-296.

Newsome WT, Britten KH, Salzman CD, Movshon JA (1990) Neuronal mechanisms of motion perception. Cold Spring Harb Symp Quant Biol 55:697-705.

Nissanov J, Eaton RC, DiDomenico R (1990) The motor output of the Mauthner cell, a reticulospinal command neuron. Brain Res 517:88-98.

Nolen TG, Hoy RR (1984) Initiation of behavior by single neurons: the role of behavioral context. Science 226:992-994.

Olberg RM (1983) Pheromone-triggered flip-flopping interneurons in the ventral nerve cord of the silkworm moth, Bombyx mori. J Comp Physiol [A] 152:297-307. 
Ramirez J-M (1988) Interneurons in the subesophageal ganglion of the locust associated with flight initiation. J Comp Physiol [A] 162:669-685.

Ritzmann RE, Pollack AJ (1994) Responses of thoracic interneurons to tactile stimulation in the cockroach, Periplaneta americana. J Neurobiol 25:1113-1128.

Salzman CD, Newsome WT (1994) Neural mechanisms for forming a perceptual decision. Science 264:231-237.

Selverston AI, Kleindienst H-U, Huber F (1985) Synaptic connectivity between cricket auditory interneurons as studied by selective photoinactivation. J Neurosci 5:1283-1292.

Smith SR, Dowd JP, Wheeler BC, Comer CM (1988) Analysis of multiunit data from a neural ensemble controlling directed movement, pp 1171-1172. Proc 10th Int Conf IEEE Eng Med Biol Soc.

Sokal RR, Rohlf FJ (1981) Biometry, 2nd Ed. San Francisco: Freeman. Stierle IE, Getman M, Comer CM (1994) Multisensory control of escape in the cockroach Periplaneta americana. I. Initial evidence from patterns of wind-evoked behavior. J Comp Physiol [A] 174:1-11.

von Helversen D, von Helversen O (1995) Acoustic pattern recognition and orientation in orthopteran insects: parallel or serial processing? J Comp Physiol [A] 177:767-774.

Westin J, Ritzmann RE, Goddard DJ (1988) Wind-activated thoracic interneurons of the cockroach. I. Responses to controlled wind stimulation. J Neurobiol 19:573-588.

Ye S, Comer CM (1993) Touch-evoked escape in Periplaneta americana: simultaneous recording of turning responses and neuronal activity in behaving animals. Soc Neurosci Abstr 19:231.

Ye S, Dowd JP, Comer CM (1995) A motion-tracking system for simultaneous recording of rapid locomotion and neural activity from an insect. J Neurosci Methods 60:199-200. 